\title{
The Influence of Topography on the Stability of the Norwegian Atlantic Current off Northern Norway
}

\author{
PEYGHAM GHAFFARI \\ Department of Arctic R\&D, Akvaplan-Niva, Oslo, Norway \\ PÅL ERIK ISACHSEN \\ Institute of Geosciences, University of Oslo, and R\&D Division, Norwegian Meteorological Institute, Oslo, Norway \\ OLE ANDERS NøST \\ Department of Arctic R\&D, Akvaplan-Niva, Trondheim, Norway \\ JAN ERIK WEBER \\ Institute of Geosciences, University of Oslo, Oslo, Norway
}

(Manuscript received 16 November 2017, in final form 13 August 2018)

\begin{abstract}
The steep continental slope off the Lofoten-Vesterålen islands of northern Norway appears to be the source of the most intense mesoscale eddy field in all of the Nordic Seas. Here we use linearized two-layer shallow-water equations to study the stability of the Norwegian Atlantic Current in this region. The study extends previous works that used linearized quasigeostrophic vertical mode equations to examine the effects of bottom topography on baroclinic instability here. We find evidence of baroclinic instability in the stacked shallow-water model but also of barotropic instability that is associated with the upper-layer lateral shear. The calculations give an indication that growth rates of barotropic instability may be comparable to or larger than those of baroclinic instability over the steepest parts of the continental slope.
\end{abstract}

\section{Introduction}

The continental slope off Norway guides the warm and salty Norwegian Atlantic Current (NwAC) through the Nordic Seas on its journey toward the Arctic Ocean. The NwAC loses heat and thereby gets denser as it flows northward along the coast. The advective heat fluxes are of major importance to the regional climate, and nutrient fluxes also impact the marine ecosystem dramatically (Skjoldal 2004; Drange et al. 2005). As it turns out, most of the cooling of the NwAC is not caused by local air-sea fluxes over the slope current itself but rather by lateral heat transport into the interior ocean basins (Isachsen et al. 2012). It is in these basins that the bulk of the heat is eventually passed onto the atmosphere, the reason

Corresponding author: Peygham Ghaffari, peygham.ghaffari@ akvaplan.niva.no being a combination of larger surface areas and longer residence times (Isachsen and Nøst 2012; Koszalka et al. 2013). Since the NwAC itself is heavily guided by the continental slope, lateral heat exchange across the slope must be facilitated by mesoscale or submesoscale eddy fluxes. Indeed, both observations and models show a mean boundary current that is also associated with enhanced eddy kinetic energy (EKE) levels (Poulain et al. 1996; Andersson et al. 2011; Koszalka et al. 2011, 2013; Søiland and Rossby 2013). Time variability of the mean current only makes up a small part of this EKE, and it is nonlinear flow features like coherent vortices-eddies-that dominate (Raj et al. 2015).

For a basic understanding of the cross-slope mixing processes that take place along the path of the NwAC one needs to know whether the primary source of the observed eddy field is barotropic or baroclinic instability or, possibly, a combination of the two. The former 
extracts kinetic energy from the mean current while the latter extracts available potential energy associated with the tilted isopycnals of a large-scale ocean current in thermal wind balance. Mysak and Schott (1977) investigated the linear stability of the NwAC off southern Norway using a quasigeostrophic (QG) two-layer model and found that the most unstable waves matched favorably with observations (Horn and Schott 1976). They concluded that the flow field along that stretch of the coast is primarily baroclinically unstable. Teigen et al. $(2010,2011)$ then investigated the stability of the West Spitsbergen Current, a branch of the NwAC in the far north. Using a linearized two-layer shallow-water (SW) model, these authors argued that the current in the north, just before it enters the Arctic, is both barotropically and baroclinically unstable.

The most intense eddy activity and lateral cooling of the NwAC is found off the Lofoten-Vesterålen islands, along a stretch of the north Norwegian coast that has a particularly narrow continental slope (Koszalka et al. 2011). The narrowing of the continental slope here intuitively suggests an enhanced lateral current shear that will be prone to barotropic instability. However, a quantitative assessment of this possibility has not been made so far. Isachsen (2015) investigated the nature of baroclinic instability off Lofoten-Vesterålen using linearized QG vertical mode calculations. The exercise suggested that the steepest part of the slope is the most baroclinically unstable (where the fastest unstable growth takes place). This result may at first appear to be inconsistent with Eady or two-layer Phillips theory, which predicts a suppression of baroclinic instability by a sloping bottom (Blumsack and Gierasch 1972; Mechoso 1980). Spall (2010) has noted, however, that high growth rates can still be found over steep topography if the isopycnals steepen accordingly. In addition, the study of Isachsen (2015) also showed fast-growing unstable modes that were associated with internal potential vorticity (PV) thickness gradients rather than with top and bottom edge waves. This last effect, which is not captured by the Eady or two-layer Phillips frameworks, ${ }^{1}$ seems to be ubiquitous along large parts of the continental slopes of the Nordic Seas and Labrador Sea (Trodahl and Isachsen 2018).

In the current manuscript we shift the perspective away from vertical mode calculations and the details of baroclinic instability and instead ask a very basic

\footnotetext{
${ }^{1}$ Internal PV thickness gradients correspond to a vertical change of isopycnal slope. Neither the Eady nor the two-layer Phillips models include such dynamics (the Phillips model would require a minimum of three layers).
}

question: could the NwAC off Lofoten-Vesterålen also be barotropically unstable? To do this we will study linear instability ${ }^{2}$ in a plane crossing the mean current, thus allowing an investigation of the joint possibility of PV gradient sign reversals both in the horizontal and vertical directions. To allow for large topographic slopes, we will also leave the QG framework and instead use two-layer SW equations. Instability of the current will still be assessed assuming wave mode solutions that are then solved for by standard eigenvalue methods.

The rest of the paper is organized as follows. In section 2 we present the linearized stability equations and the numerical solution method used. Then, in section 3 , we present the results obtained by solving those equations for various shelf slopes. We thus study the growth dependency on the topographic steepness and use various measures, including energy transfer terms, to determine whether baroclinic or barotropic instability is at play. The paper ends with a brief discussion of the results and conclusions in section 4 .

\section{Model formulation}

\section{a. The linearized two-layer shallow-water model}

The NwAC is a surface-intensified baroclinic jet of width $40-80 \mathrm{~km}$ that is strongly guided by the continental slope below (Mauritzen 1996; Orvik and Niiler 2002). The jet widens and narrows depending on the steepness of the slope below, and over the very steep slope off the Lofoten-Vesterålen islands the current accelerates to attain surface velocities in excess of $0.5 \mathrm{~m} \mathrm{~s}^{-1}$ (Koszalka et al. 2011). There is time variability involved, especially at the seasonal time scale where current speeds in winter are almost twice as large as speeds in summer (Orvik and Skagseth 2003; Skagseth et al. 2004; Mork and Skagseth 2010). A stability assessment could take account of this time dependence, for example, by comparing stability during summer and winter months independently. But for our very basic question of whether the current is in fact barotropically unstable (in addition to being baroclinically unstable), we start by an analysis of time-mean conditions.

Figure 1 shows the bathymetry in the region as well as the hydrography and flow field extracted from an 800-m-resolution operational regional ocean model operated by the Norwegian Meteorological Institute (Albretsen et al. 2011). Figure 1c shows representative conditions when model data have been averaged over

\footnotetext{
${ }^{2}$ We only investigate the possibility of linear instability here. A nonlinear system may also become unstable under finite-amplitude perturbations even if the linearized version of the dynamics remains stable (Butler and Farrell 1992).
} 


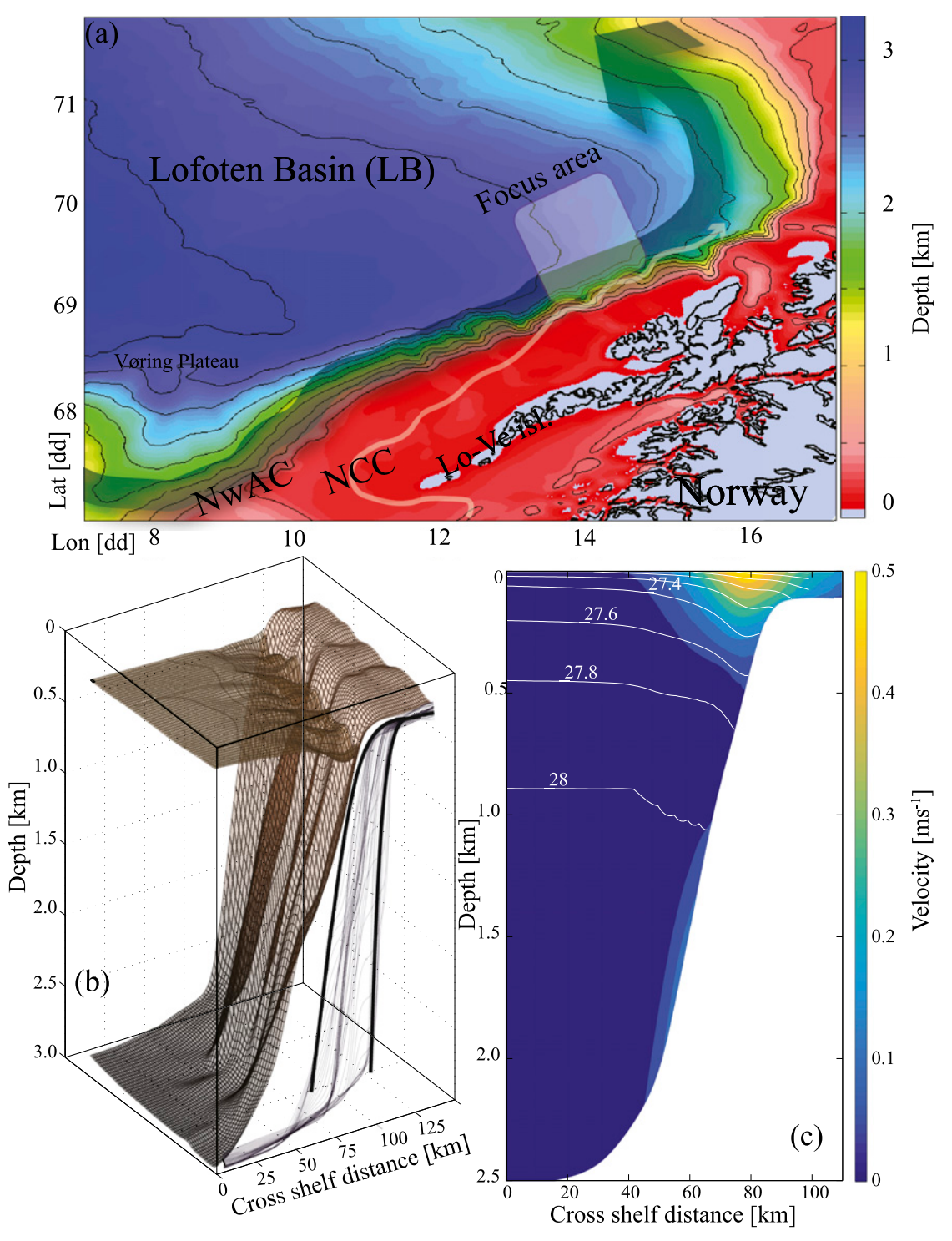

FIG. 1. The bathymetry and time-mean flow field off the Lofoten-Vesterålen islands: (a) overview map with the focus region highlighted, (b) the bathymetry along the focus region and exponential fits (black lines) covering the range of bottom slopes, and (c) time-mean alongshore flow velocity (colors) and potential density field (contours) from an $800-\mathrm{m}$ resolution regional ocean model of the region (Albretsen et al. 2011).

one year (2009-10) and over several cross-slope transects (gray region in Fig. 1a). The model is in agreement with observations in revealing a surface-intensified baroclinic jet that reaches $0.5 \mathrm{~m} \mathrm{~s}^{-1}$ and has a width of $50-60 \mathrm{~km}$. The current extends down approximately to the $\sigma_{\theta}=27.7 \mathrm{~kg} \mathrm{~m}^{-3}$ isopycnal, more or less the bottom of the Atlantic Water layer (Mauritzen 1996; Isachsen et al. 2007). This bounding isopycnal then intersects the continental slope underneath the jet maximum at a depth of a few hundred meters. A weaker current (speed less than $0.1 \mathrm{~m} \mathrm{~s}^{-1}$ ) below 1000-m depth transports dense waters around the Nordic Seas (Käse et al. 2009).
To operate with self-consistent fields, we use the timeaveraged hydrography and currents from the operational model (Fig. 1c) to form the basis for our two-layer linearized SW model of the region. We use a local Cartesian coordinate system where the $x$ axis is directed toward the coast, the $y$ axis is directed along the shore, and the vertical $z$ axis is positive upward-as sketched in Fig. 2. In the absence of motion the thicknesses of the two layers are $H_{1}$ and $H_{2}$, where the lower-layer thickness can vary in the $x$ direction due to bottom topography. When motion in the two layers is included the thicknesses are 


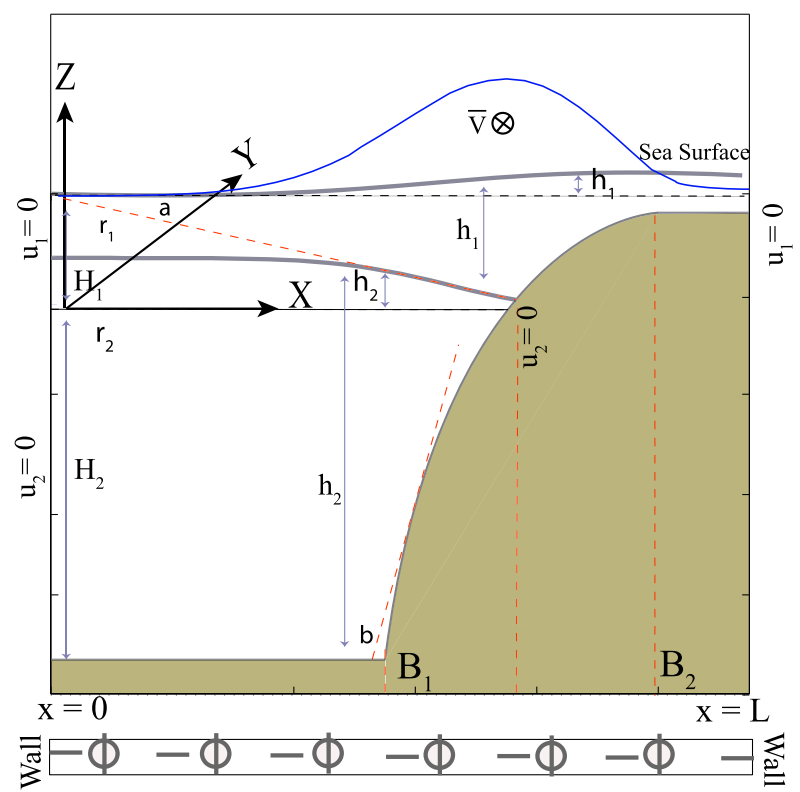

FIG. 2. Configuration of our idealized two-layer shallow-water model. Horizontal lines along the bottom denote $u$ grid points while circles with vertical lines denote $\eta$ and $v$ grid points.

$$
\begin{aligned}
& h_{1}=H_{1}+\eta_{1}-\eta_{2}, \quad \text { and } \\
& h_{2}=H_{2}+\eta_{2},
\end{aligned}
$$

where $\eta_{1}$ is the dynamical free surface displacement (above $z=0$ ) and $\eta_{2}$ is the dynamical displacement of the interface that separates the two layers (above $z=-H_{1}$ ). The sea surface and interface displacements are related to horizontal velocities $\mathbf{u}_{1}=$ $u_{1} \hat{\mathbf{i}}+v_{1} \hat{\mathbf{j}}$ (top layer) and $\mathbf{u}_{2}=u_{2} \hat{\mathbf{i}}+v_{2} \hat{\mathbf{j}}$ (bottom layer) by the shallow-water momentum and continuity equations

$\frac{\partial \mathbf{u}_{1}}{\partial t}+\mathbf{u}_{1} \cdot \nabla \mathbf{u}_{1}+f \hat{\mathbf{k}} \times \mathbf{u}_{1}=-g \nabla \eta_{1}$,

$\frac{\partial}{\partial t}\left(\eta_{1}-\eta_{2}\right)=-\nabla \cdot\left(\mathbf{u}_{1} h_{1}\right)$

$\frac{\partial \mathbf{u}_{2}}{\partial t}+\mathbf{u}_{2} \cdot \nabla \mathbf{u}_{2}+f \hat{\mathbf{k}} \times \mathbf{u}_{2}=-g \nabla \eta_{1}-g^{\prime} \nabla \eta_{2}, \quad$ and

$\frac{\partial \eta_{2}}{\partial t}=-\nabla \cdot\left(\mathbf{u}_{2} h_{2}\right)$.

Here $f$ is the Coriolis parameter, $g$ is the gravitational acceleration, and $g^{\prime}=g \Delta \rho / \rho_{0}$ is the reduced gravity for density jump $\Delta \rho=\rho_{2}-\rho_{1}$ between the two layers and reference density $\rho_{0}$. We linearize the system around a geostrophically balanced mean flow following the coast. This background flow is allowed to vary in the $x$ direction and between the two layers. So we write

$$
\begin{aligned}
& f \bar{v}_{1}=g \frac{\partial \bar{\eta}_{1}}{\partial x}, \quad \text { and } \\
& f \bar{v}_{2}=g \frac{\partial \bar{\eta}_{1}}{\partial x}+g^{\prime} \frac{\partial \bar{\eta}_{2}}{\partial x},
\end{aligned}
$$

where $\bar{\eta}_{1}$ and $\bar{\eta}_{2}$ are the mean sea surface and interface displacements balancing the Coriolis acceleration of mean flows $\bar{v}_{1}$ and $\bar{v}_{2}$. Now we allow for wavelike perturbations around this mean flow with an amplitude scale $\epsilon$ that is small compared to the mean flow itself. The variables are then decomposed into basic state parts and perturbations,

$$
\left[\eta_{j}, u_{j}, v_{j}\right]=\left[\bar{\eta}_{j}, 0, \bar{v}_{j}\right]+\epsilon\left[\eta_{j}^{\prime}, u_{j}^{\prime}, v_{j}^{\prime}\right]+O\left(\epsilon^{2}\right),
$$

where the primed quantities are the perturbations. The linearized equations for the perturbations are (in component form)

$$
\begin{aligned}
& \frac{\partial u_{1}^{\prime}}{\partial t}+\bar{v}_{1} \frac{\partial u_{1}^{\prime}}{\partial y}-f v_{1}^{\prime}=-g \frac{\partial \eta_{1}^{\prime}}{\partial x} \\
& \frac{\partial v_{1}^{\prime}}{\partial t}+u_{1}^{\prime} \frac{\partial \bar{v}_{1}}{\partial x}+\bar{v}_{1} \frac{\partial v_{1}^{\prime}}{\partial y}+f u_{1}^{\prime}=-g \frac{\partial \eta_{1}^{\prime}}{\partial y}
\end{aligned}
$$

$\frac{\partial}{\partial t}\left(\eta_{1}^{\prime}-\eta_{1}^{\prime}\right)=-\frac{\partial}{\partial x}\left(u_{1}^{\prime} \bar{h}_{1}\right)-\bar{h}_{1} \frac{\partial v_{1}^{\prime}}{\partial y}-\bar{v}_{1} \frac{\partial}{\partial y}\left(\eta_{1}^{\prime}-\eta_{2}^{\prime}\right)$,

$$
\frac{\partial u_{2}^{\prime}}{\partial t}+\bar{v}_{2} \frac{\partial u_{2}^{\prime}}{\partial y}-f v_{2}^{\prime}=-g \frac{\partial \eta_{1}^{\prime}}{\partial x}-g^{\prime} \frac{\partial \eta_{2}^{\prime}}{\partial x}
$$

$$
\frac{\partial v_{2}^{\prime}}{\partial t}+u_{2}^{\prime} \frac{\partial \bar{v}_{2}}{\partial x}+\bar{v}_{2} \frac{\partial v_{2}^{\prime}}{\partial y}+f u_{2}^{\prime}=-g \frac{\partial \eta_{2}^{\prime}}{\partial y}-g^{\prime} \frac{\partial \eta_{2}^{\prime}}{\partial y}, \quad \text { and }
$$

$\frac{\partial \eta_{2}^{\prime}}{\partial t}=-\frac{\partial}{\partial x}\left(u_{2}^{\prime} \bar{h}_{2}\right)-\bar{h}_{2} \frac{\partial v_{2}^{\prime}}{\partial y}-\bar{v}_{2} \frac{\partial \eta_{2}^{\prime}}{\partial y}$,

where the first-order perturbation terms, that is, $O(\epsilon)$ with $\epsilon \ll 1$, have been retained, while the nonlinear perturbation terms $O\left(\epsilon^{2}\right)$ are neglected.

We look for perturbation solutions that are wavelike in the $y$ direction and write

$$
\left[u_{j}^{\prime}, v_{j}^{\prime}, \eta_{j}^{\prime}\right]=\operatorname{Re}\left\{\left[\hat{u}_{j}(x), \hat{v}_{j}(x), \hat{\eta}_{j}(x)\right] e^{i(l y-\omega t)}\right\}
$$

for each of the two layers $(j=1,2)$. Here $l$ and $\omega$ are the wavenumber and wave frequency, respectively, and $\hat{u}_{j}(x), \hat{v}_{j}(x)$, and $\hat{\eta}_{j}(x)$ are horizontal structure functions in the $x$ direction. With this ansatz, inserting the perturbation form (16) into the linearized equations (10)-(15) yields 
$g \frac{d \hat{\eta}_{1}}{d x}+i l \bar{v}_{1} \hat{u}_{1}-f \hat{v}_{1}=i \omega \hat{v}_{1}$

$i g l \hat{\eta}_{1}+\left(f+\frac{d \bar{v}_{1}}{d x}\right) \hat{u}_{1}+i l \bar{v}_{1} \hat{v}_{1}=i \omega \hat{v}_{1}$,

$\frac{d}{d x}\left(\bar{h}_{1} \hat{u}_{1}+\bar{h}_{2} \hat{u}_{2}\right)+i l\left(\bar{v}_{2}-\bar{v}_{1}\right) \hat{\eta}_{2}$

$+i l\left(\bar{h}_{2} \hat{v}_{2}+\bar{h}_{1} \hat{v}_{1}+\bar{v}_{1} \hat{\eta}_{1}\right)=i \omega \hat{\eta}_{1}$,

$g \frac{d \hat{\eta}_{1}}{d x}+g^{\prime} \frac{d \hat{\eta}_{2}}{d x}+i l \bar{v}_{2} \hat{u}_{2}-f \hat{v}_{2}=i \omega \hat{u}_{2}$

$i l\left(g \hat{\eta}_{1}+g^{\prime} \hat{\eta}_{2}+\bar{v}_{2} \hat{v}_{2}\right)+\hat{u}_{2}\left(f+\frac{d \bar{v}_{2}}{d x}\right)=i \omega \hat{v}_{2}, \quad$ and

$i l\left(\bar{v}_{2} \hat{\eta}_{2}+\bar{h}_{2} \hat{v}_{2}\right)+\frac{d}{d x}\left(\bar{h}_{2} \hat{u}_{2}\right)=i \omega \hat{\eta}_{2}$,

where the continuity equation for the bottom layer has been substituted into that for the top layer.

The above equations are discretized on a staggered grid in the $x$ direction, with $u$ points halfway between $\eta / v$ points as shown in Fig. 2. The grid spacing used is $800 \mathrm{~m}$, the same as used in the primitive equation model. We use centered differences so that, for example, the last two equations (for $\hat{v}_{2}$ and $\hat{\eta}_{2}$ ) become

$i l\left(g \hat{\eta}_{1, j}+g^{\prime} \hat{\eta}_{2, j}+\bar{v}_{2, j} \hat{v}_{2, j}\right)+\hat{u}_{2, j}^{(\eta / v)}\left(f+\frac{d \bar{v}_{2}}{d x_{j}}\right)=i \omega \hat{v}_{2, j}$,

and

$$
i l\left(\bar{v}_{2, j} \hat{\eta}_{2, j}+\bar{h}_{2, j} \hat{v}_{2, j}\right)+\frac{1}{\delta x}\left(\bar{h}_{2, j}^{(u)} \hat{u}_{2, j}-\bar{h}_{2, j-1}^{(u)} \hat{u}_{2, j-1}\right)=i \omega \hat{\eta}_{2, j},
$$

where $\delta x$ is the grid spacing. Variables here are by default evaluated at their native grid points (either $\eta / v$ points or $u$ points), and we use superscripts in the above expressions to indicate where interpolation onto the other variable's grid points has been done. Making these interpolations explicit gives

$$
\begin{aligned}
& i l\left(g \hat{\eta}_{1, j}+g^{\prime} \hat{\eta}_{2, j}+\bar{v}_{2, j} \hat{v}_{2, j}\right)+\left(\frac{\hat{u}_{2, j-1}+\hat{u}_{2, j}}{2}\right)\left(f+\frac{d \bar{v}_{2}}{d x_{j}}\right) \\
& \quad=i \omega \hat{v}_{2, j}, \text { and } \\
& i l\left(\bar{v}_{2, j} \hat{\eta}_{2, j}+\bar{h}_{2, j} \hat{v}_{2, j}\right)+\frac{1}{\delta x}\left[\left(\frac{\bar{h}_{2, j}+\bar{h}_{2, j+1}}{2}\right) \hat{u}_{2, j}\right. \\
& \left.\quad-\left(\frac{\bar{h}_{2, j-1}+\bar{h}_{2, j}}{2}\right) \hat{u}_{2, j-1}\right]=i \omega \hat{\eta}_{2, j} .
\end{aligned}
$$

Finally, the above equation set requires lateral boundary conditions. We impose impermeable vertical walls at $x=0$ in the west and $x=L$ in the east. This implies kinematic boundary conditions $\hat{u}_{1}=\hat{u}_{2}=0$ at $x=0$ and $\hat{u}_{1}=0$ at $x=L$. Assuming a wall in the upper layer in the east is reasonable (modeling the coast). There is, of course, no justification for assuming a wall condition in the west, and the impact of this will have to be assessed a posteriori, that is, by inspecting the amplitude of the solution near the western boundary.

The boundary condition for the lower layer in the east is the more problematic one. As shown in Fig. 2 we consider a situation in which the interface between the two layers intersects or "incrops" onto the continental slope in the east. The correct linearized kinematic boundary condition for this problem is derived in appendix A. It turns out to be equivalent to the volume conservation equation for the lower layer [(15) and (22)] but with mean layer depth $\bar{h}_{2}$ equal to zero. Assuming $\hat{u}_{2}=0$ here as well is only formally correct in the limit of infinite bottom slope (a vertical wall).

Previous studies have circumvented this issue by picking an isopycnal light enough that it runs above the continental slope and thus extends all the way to the coastal wall (Teigen et al. 2011; Poulin et al. 2014), or they have made the bottom slope vertical at the depth of the isopycnal incrop (Mysak and Schott 1977). Then, obviously, $\hat{u}_{2}=0$ would apply. For our problem, however, we pick a dividing isopycnal that incrops onto a nonvertical continental slope (as suggested by Fig. 1). Hence, setting $\hat{u}_{2}=0$ in the east is, strictly speaking, not correct. However, calculations made (see appendix A) suggest that the overall qualitative behavior of the unstable modes of our problem is remarkably insensitive to the choice made for this lower-layer boundary condition. So in the following we proceed with the assumption $\hat{u}_{2}=0$ at the incrop point.

The resulting equations form a standard matrix eigenvalue problem in $\boldsymbol{\chi}_{j}=\left[\hat{u}_{j}, \hat{v}_{j}, \hat{\eta}_{j}\right]$,

$$
\mathbf{M}_{i j} \boldsymbol{\chi}_{j}=\omega \boldsymbol{\chi}_{j}
$$

where the two-dimensional matrix $\mathbf{M}_{i j}$ contains the coefficients in the centered finite-difference representation of (17)-(22) with kinematic boundary conditions implemented. The wavenumber $l$ is a parameter to be varied while the complex eigenvalue $\omega=\omega_{r}+i \omega_{i}$ is part of the solution, determining the phase velocity $c_{r}=\omega_{r} / l$ and growth rate $\omega_{i}$ of the modes. For $\omega_{i}>0$ a given wave is unstable. We tested our discretization scheme by successfully reproducing results reported by Teigen et al. (2011) and Poulin et al. (2014). 


\section{b. Idealized geometry and flow field for the Lofoten-Vesterålen region}

Figure $1 \mathrm{c}$ shows that the continental slope connects a shelf region of depth less than $200 \mathrm{~m}$ to an ocean basin of depth around $2.5 \mathrm{~km}$. In keeping the model as simple as possible, we made an idealized exponential shelf profile of form

$$
H(x)=\left\{\begin{array}{cl}
H_{\max }, & 0 \leq x<B_{1}, \\
H_{\min } \exp \left[b\left(B_{1}-x\right)\right], & B_{1} \leq x<B_{2}, \\
H_{\min }, & B_{2} \leq x \leq L .
\end{array}\right.
$$

Here $H_{\min }=180 \mathrm{~m}$ is the water depth over the continental shelf, $H_{\max }=2500 \mathrm{~m}$ is the depth over the deep basin, $B_{1}$ and $B_{2}$ are the position of the western and eastern extents of the slope, and, finally, $b$ is a positive constant describing the steepness of the slope. For shelf width $B=B_{2}-B_{1}$ this becomes

$$
b=B^{-1} \ln \frac{H_{\max }}{H_{\min }} .
$$

The shelf width $B$ will be varied in what follows from about 10 to $120 \mathrm{~km}$. We note that this topography has discontinuous derivatives at two points. However, sensitivity tests that involved smoothed versions of the topography showed that these discontinuities have negligible effects on growth rates.

We then specified a simplified jet that approximately fits the surface jet seen in Fig. 1c:

$$
\bar{v}_{j}(x)=V_{j} \frac{x}{B_{\text {jet }}} \exp \left(\frac{x-x_{\text {jet }}}{B_{\text {jet }}}\right)^{2} .
$$

Here $V_{j}$ is the maximum jet velocity in layer $j, x_{\text {jet }}$ is the $x$ position of this maximum, and $B_{\text {jet }}$ gives the half-width of the jet. In what follows, we consider a jet with a typical width of $2 B_{\text {jet }}=60 \mathrm{~km}$ and an upper-layer core velocity of $V_{1}=0.45 \mathrm{~m} \mathrm{~s}^{-1}$. Below we will mostly study unstable growth for a lower layer at rest $\left(V_{2}=0\right)$, but some calculations were also made for weak lower-layer flow.

The two layers were fitted to the geometry defined by the $\sigma_{\theta}=27.7 \mathrm{~kg} \mathrm{~m}^{-3}$ isopycnal in Fig. 1c. A reduced gravity was first estimated from differences in areaaveraged model densities above and below this isopycnal $\left(g^{\prime}=8 \times 10^{-3} \mathrm{~m} \mathrm{~s}^{-2}\right)$. Then the position of the interface between the two layers was integrated from the thermal wind relations, starting at an initial depth of $250 \mathrm{~m}$ far offshore in the west. The remaining parameters needed to specify our two-layer SW model were $f=1.4 \times 10^{-4} \mathrm{~s}^{-1}$ and $g=9.8 \mathrm{~m} \mathrm{~s}^{-2}$. With these parameters our model has an internal deformation radius
$R_{d}=\sqrt{g^{\prime} H_{\text {eff }} / f}$ [with $\left.H_{\text {eff }}=H_{1} H_{2} /\left(H_{1}+H_{2}\right)\right]$ of around $10 \mathrm{~km}$, a value in good agreement with estimates made by Nurser and Bacon (2014, their Fig. 2).

\section{Results}

In the following we investigate linear unstable growth in the two-layer shallow-water model as a function of variable continental slope steepness. The slope steepness impacts instability by changing the thickness PV gradients near the bottom. In the modified Eady model and two-layer Phillips model of Blumsack and Gierasch (1972) and Mechoso (1980), it is not the bottom slope alone but rather the ratio between bottom slope and isopycnal slope that governs stability. So for bottom slope $\beta$ and isopycnal slope $\alpha$, the slope parameter is

$$
\text { To } \equiv \beta / \alpha .
$$

For the situation along the Norwegian continental slope where the isopycnal and bottom slopes have different signs (Figs. 1,2), To is negative. For this configuration the theory predicts both reduced growth rates and length scales compared to the equivalent flat-bottom configuration. Below we will see whether this QG prediction carries over to the SW system, which is more appropriate for large topographic and isopycnal slopes. But our main objective, as mentioned above, is to see whether barotropic instability due to the lateral shear in the surface-intensified current may also be present off Lofoten-Vesterålen.

\section{a. Growth rates as a function of To}

We varied To by changing the bottom slope while keeping the jet strength and its vertical shear (hence isopycnal slope) fixed. Most calculations were based on the configuration where the lower layer is at rest (what we call a "passive" lower layer), but the effect of a nonzero lower layer flow ("active" lower layer) was also investigated briefly. Figure 3 shows growth rates of unstable modes for our base case, using bounding isopycnal $\sigma_{\theta}=27.7 \mathrm{~kg} \mathrm{~m}^{-3}$, a passive lower layer, and three different values of To. For comparison with modified Eady and two-layer Phillips theory, the growth rates in the plot have been normalized by $V_{0} / R_{d}$, where $V_{0}=$ $\left|\bar{v}_{1}-\bar{v}\right|_{2}$ is the velocity jump between the layers, and $R_{d}$ is the internal deformation radius (see above). For the same reason, wavenumbers have been normalized by $l_{d}=1 / R_{d}$.

Many unstable waves exist, but three modes dominate. We label these modes $\mathrm{A}, \mathrm{B}$, and $\mathrm{C}$ according to their lateral scale, with modes $\mathrm{A}$ and $\mathrm{C}$ having the smallest and largest scales, respectively. Mode C is 

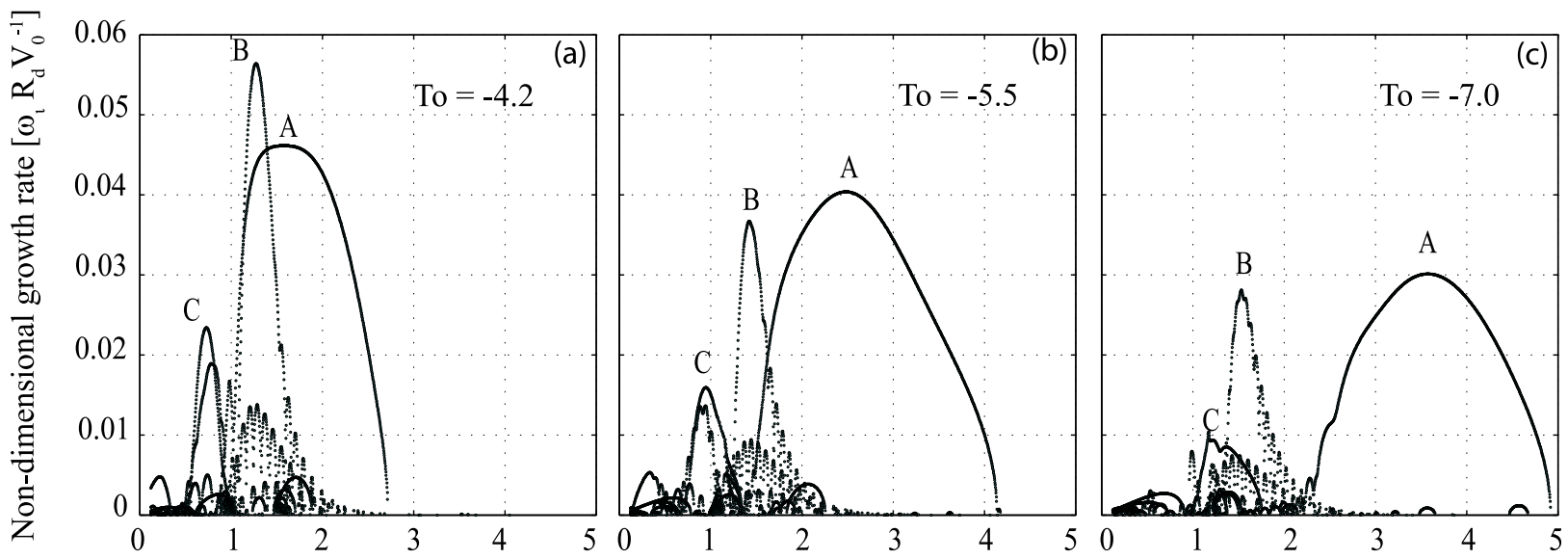

Non-dimensional wave number $\left[\mathrm{R}_{\mathrm{d}} \mathrm{l}_{\max }\right]$

FIG. 3. Nondimensional growth rate as functions of nondimensional wavenumber for the base case of bounding isopycnal $\sigma_{\theta}=$ $27.7 \mathrm{~kg} \mathrm{~m}^{-3}$. Three values for the slope parameter are compared: (a) To $=-4.2$, (b) To $=-5.5$, and (c) To $=-7.0$. Three dominant unstable modes are labeled (A, B, and C).

really a pair of modes that follow each other closely as To is varied. As it turns out, all three modes show agreement with the modified Eady model of Blumsack and Gierasch (1972): they all have a long-wave cutoff (absent for a flat bottom) and they all grow slower and attain smaller scales as the magnitude of the topographic slope grows relative to that of the isopycnal slope. In fact, for very steep bottom slopes the magnitude of mode $\mathrm{C}$ drops to levels comparable with the general "noise level" of other unstable waves. But all three modes remain intact over the range of slope parameters tested here, and modes A and B always dominate.

Figure 4 shows the behavior over a larger range of To. Shown are maximum growth rates and corresponding wavenumber (both nondimensionalized) for each of the three modes as functions of To. The tendencies noted above are seen to be general, that is, a decrease in both growth rates and lateral scales for steeper bottom slopes-both in agreement with modified Eady theory. Here we show the results for both a passive lower layer $\left(\bar{v}_{2}=0\right)$ and an active lower layer in which $\bar{v}_{2}=0.2 \bar{v}_{1}$. Introducing a mean flow in the lower layer does not impact the results qualitatively but leads to somewhat larger nondimensional growth rates. Presumably, the change in growth rates reflects quantitative discrepancies between the SW and QG frameworks for steep slopes.

So a steeper bottom slope influences growth for all three unstable modes in qualitatively similar ways. Nevertheless, there are subtle differences. For both a passive and active lower layer the scales of modes A and C show a strong sensitivity to To. This sensitivity is much smaller for mode B. Conversely, the growth rate of mode B is more sensitive to To than the other two modes, and whereas this is the fastest-growing mode for weak bottom slopes, it grows slower than mode A for steep slopes. These are indications that the dynamics of mode B might be distinct from that of modes A and C. We redid the above calculations using a range of different bounding isopycnals, with corresponding differing layer depths and reduced gravities. Growth rates and length scales differed in detail (see an example in Fig. 5), but the three modes and their qualitative response to changes in To remained throughout these calculations. As for the reference case shown here, mode B decays more rapidly with increasing bottom slope than modes $\mathrm{A}$ and $\mathrm{C}$.

\section{b. Spatial structures of unstable modes}

The spatial structures of the three modes, for slope parameter To $=-5.5$, are shown in Fig. 6. We have plotted the real part of the sea surface displacement, interface displacement, and all velocity components. The color scales are arbitrary, but the magnitudes of variables of the same type can be compared to each other. Before looking into the details, it is worth noting that all modes have vanishing structure in the vicinity of the western boundary. Hence, our using the wall assumption $\left(u^{\prime}=0\right)$ at what should really be an open boundary in the west appears to be justifiable.

Mode B exists only over regions with two layers, disappearing east of the isopycnal incrop point. It also has bottom-intensified cross-shelf $(u)$ velocities, particularly near the bottom slope where the lower layer is thinner than the upper layer. This is to be expected from a baroclinic instability process that tends to flatten the 

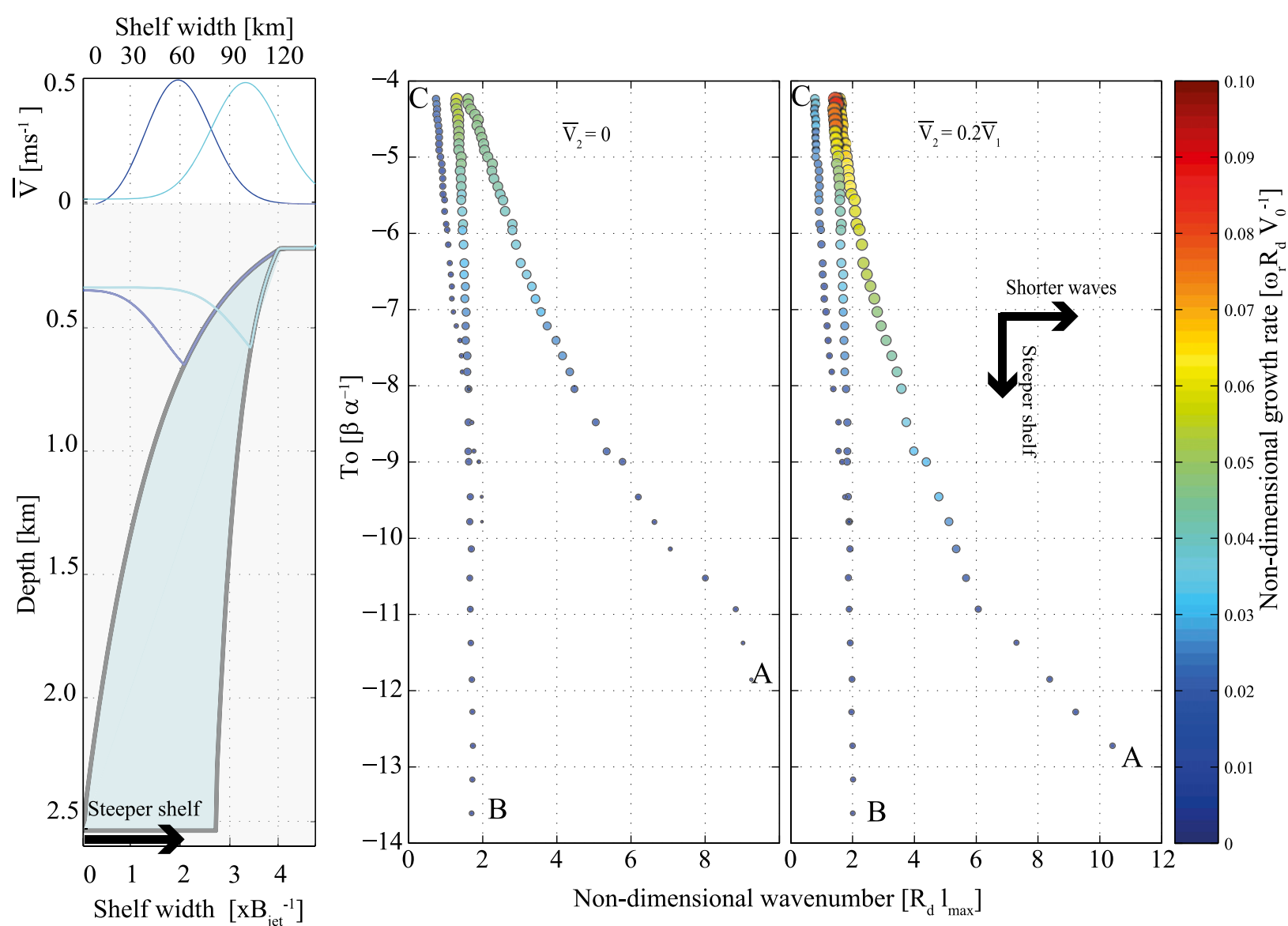

FIG. 4. (left) An illustration of how To is varied by changing the steepness of the topographic slope (the $x$ coordinate has been scaled by jet width $B_{\text {jet }}$ ). The nondimensional growth rates and corresponding nondimensional wavenumbers for the three fastest-growing modes for various values of To: (center) passive lower layer $\left(\bar{v}_{2}=0\right)$ and (right) active lower layer $\left(\bar{v}_{2}=0.2 \bar{v}_{1}\right)$.

isopycnal by bolus transports that have opposite signs in the two layers. For equal but opposite layer transports, a thinner lower layer requires a correspondingly higher bolus velocity. A larger velocity perturbation in the lower layer is also predicted, from dynamic considerations, by the modified Eady model when the slope parameter To is negative, as is the case here (see, e.g., Fig. 6 in Isachsen 2015).
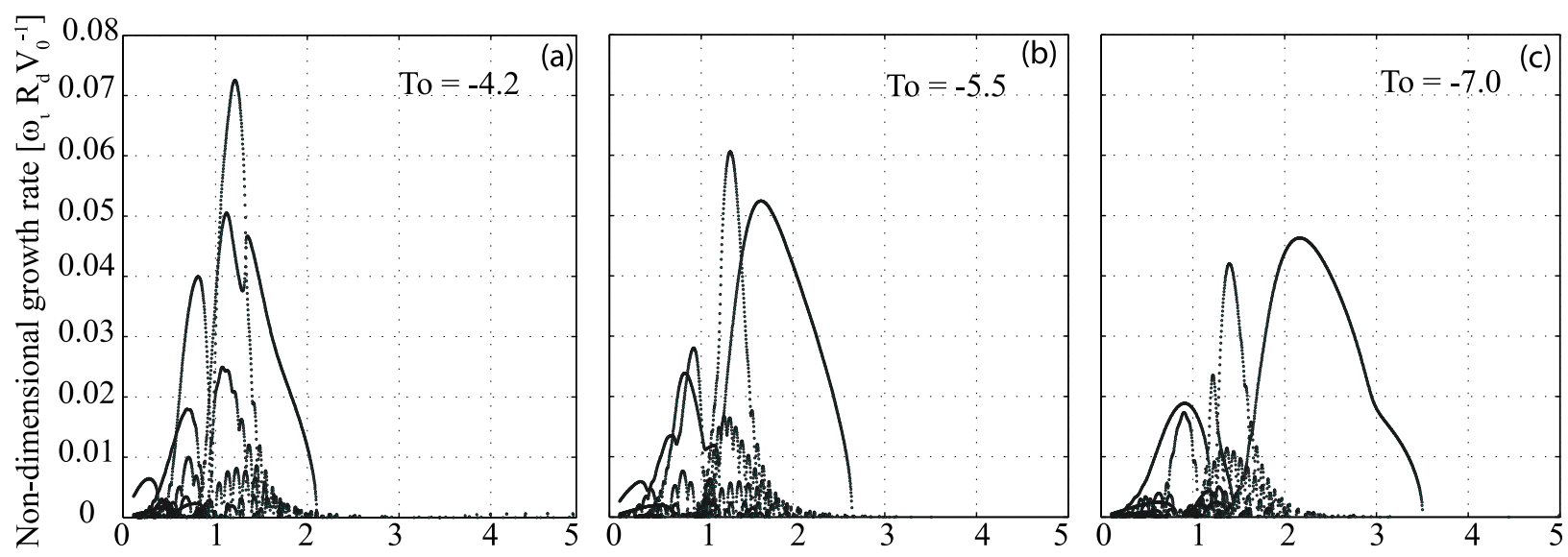

Non-dimensional wave number $\left[\mathrm{R}_{\mathrm{d}} 1_{\max }\right]$

FIG. 5. As in Fig. 3, but based on bounding isopycnal $\sigma_{\theta}=27.6 \mathrm{~kg} \mathrm{~m}^{-3}$. 

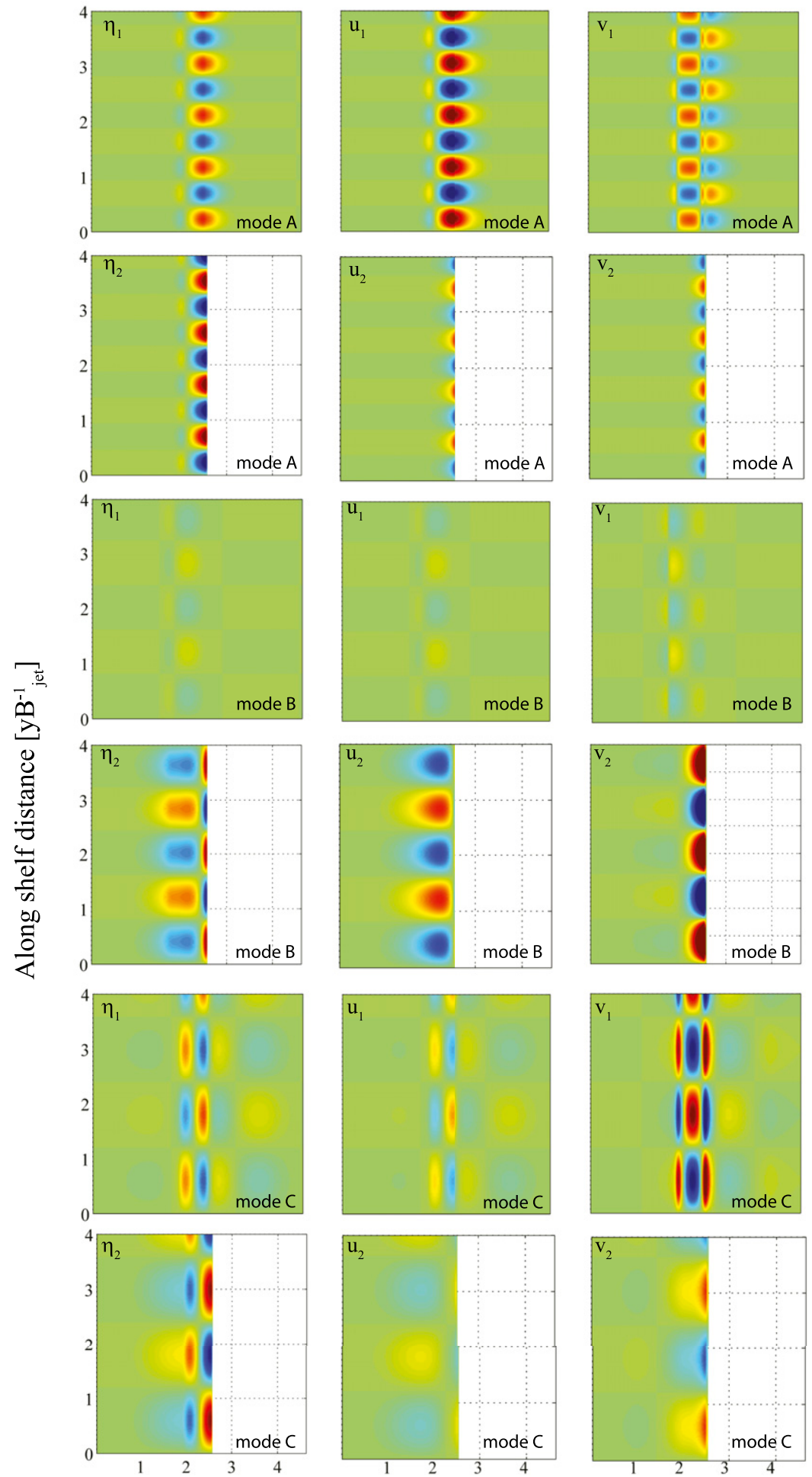

Cross shelf distance $\left[\mathrm{xB}^{-1}{ }_{\text {jet }}\right]$

FIG. 6. The spatial structure of modes A, B, and C (extracted from the peaks of the growth curves) for To $=-5.5$. The six top panels show mode $\mathrm{A}$, the middle six show mode $\mathrm{B}$, and the lower six show mode $\mathrm{C}$. Color scales are arbitrary but are constant for similar variables. The exception is the sea surface displacement $\eta_{1}$, which has been divided by $g^{\prime} / g$ so that its dynamic effect on layer 2 can be compared with that of the interface displacement $\eta_{2}$. 


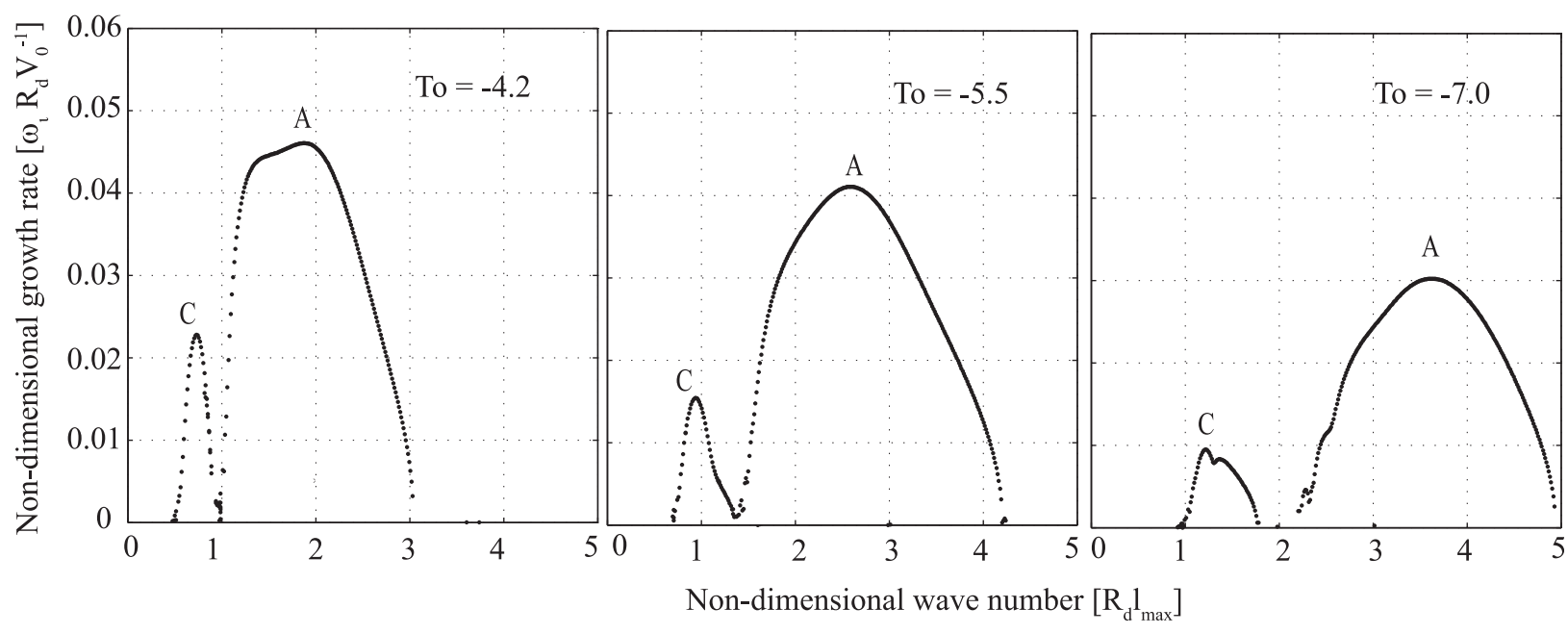

FIG. 7. Growth in the one-layer model. Nondimensional growth rate as functions of nondimensional wavenumber for variable shelf steepness: (left) To $=-4.2$, (center) To $=-5.5$, and (right) To $=-7.0$.

Modes A and C, in contrast, have characteristics that are not obviously consistent with baroclinic instability. For one, their surface-layer manifestation extends east of the intersect point, into the region where only one layer exists. They are also surface-intensified near the slope, in contradiction to the expected vertical structure of baroclinic instability for a thinner lower than upper layer near the intersect point and for To $<0$.

\section{c. Checking for evidence of barotropic instability in the top layer}

The examination of the mode structure above is qualitative and inconclusive, but there is an indication that mode $\mathrm{B}$ reflects baroclinic instability whereas modes $\mathrm{A}$ and $\mathrm{C}$ do not, at least not in its pure form. All modes may of course reflect some form of mixed baroclinic-barotropic instability, but here we will first explore the two distinct limits. Thus, to check whether modes $\mathrm{A}$ and $\mathrm{C}$ reflect barotropic instability acting on the lateral velocity shear in the top layer, we did an eigen calculation where we fixed the interface position, thus making the lower layer dynamically inactive. In this configuration the interface acts as a rigid lower boundary, an "ocean bottom" for the top layer west of the intersect point. In such a "thought experiment" baroclinic instability, if originally present, would be eliminated whereas barotropic instability acting in the top layer would prevail. Growth rates and scales of a barotropic instability mode might be quantitatively affected by a rigid lower interface, but the underlying source of barotropic instability, the lateral velocity shear, would be unaffected.

Results of these new calculations, now only involving variables $u_{1}, v_{1}$, and $\eta_{1}$, are shown in Fig. 7 for the same To values as used in Fig. 3. We detect two growing modes that corresponded to the original modes $\mathrm{A}$ and $\mathrm{C}$. The growth rates and scales of the modes, in fact, are only slightly modified from the original two-layer calculations. So modes A and C basically remain intact in the one-layer calculation, strongly suggesting that they are lateral shear or barotropic instabilities. In contrast, mode B has disappeared completely, again confirming that this mode requires two layers and reflects baroclinic instability.

Another indication that lateral shear instability takes place in the top layer can be found by examining the Rayleigh-Kuo integral constraint for this layer (CushmanRoisin and Beckers 2011). A necessary condition for instability is that the layer PV gradient

$$
Q_{x}=\frac{\partial}{\partial x}\left(\frac{f+\bar{\zeta}_{1}}{\bar{h}_{1}}\right),
$$

changes sign somewhere in the domain (here $\bar{h}_{1}=$ $H_{1}+\bar{\eta}_{1}-\bar{\eta}_{2}$ is the upper layer thickness with geostrophic mean flow included and $\bar{\zeta}_{1} \equiv \partial \bar{v}_{1} / \partial x$ is the meanflow relative vorticity). Figure 8 shows this lateral PV gradient in the upper layer for a range of To values, that is, for a range of bottom slopes. The calculation shows two zero crossings for all To configurations, in agreement with there being two unstable modes (A and C). Note that the topography is smoothed to avoid discontinuity in the PV gradient at the transition points (at the interface incrop and where our idealized bottom goes from being an exponential to being flat). The calculation thus shows that the upper-layer relative vorticity gradient is large enough to trigger barotropic instability and that the layer thickness gradient is not able to avoid PV-gradient reversals. Apparently, this holds true for a wide range of 


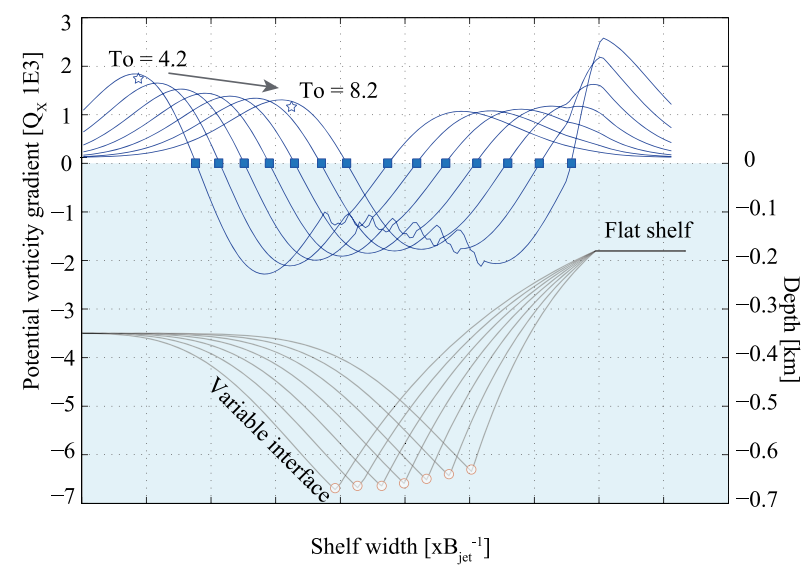

FIG. 8. The lateral PV gradient in the upper layer for a range of To values. Blue squares show where the PV gradient changes sign. Also shown is the "bottom" (the interface) felt by the upper layer for the same To values. The upper-layer bottom has been smoothed with a 10-point running-average filter.

bottom topographic slopes that we have considered to be defining the possible realistic parameter range for the steep slope off Lofoten-Vesterålen.

\section{d. Energy conversions}

As a final look into the nature of the various unstable modes found here, we look at their energy conversion rates. Pure baroclinic instability extracts mean available potential energy (MAPE) into eddy available potential energy (EAPE) while barotropic instability extracts mean kinetic energy (MKE) into EKE. A mixed baroclinic-barotropic instability process may then be defined as one that extracts both MAPE and MKE from the mean flow (Olbers et al. 2012).

To second order in perturbation quantities, volumeintegrated conversion terms corresponding to extraction of MAPE and MKE (with mean flow only in the top layer) are (see appendix B)

$$
C_{\mathrm{MAPE} \rightarrow \mathrm{EAPE}}=\left\langle g\left(\bar{v}_{1}-\bar{v}_{2}\right) \overline{\eta_{1}^{\prime} \partial \eta_{2}^{\prime} / \partial y}\right\rangle,
$$

and

$$
C_{\mathrm{MKE} \rightarrow \mathrm{EKE}}=-\left\langle\bar{h}_{1} \overline{u_{1}^{\prime} v_{1}^{\prime}} \frac{\partial \bar{v}_{1}}{\partial x}\right\rangle
$$

where the overbars over perturbation quantities, and the angle brackets indicate integrals over $y$ and $x$, respectively. MAPE is extracted from the upper-layer flow if the area-integrated form drag on the interface is positive. MKE is extracted if Reynolds momentum fluxes in the top layer are, on average, down the mean velocity gradient, that is, out of the jet.

We study the situation for a moderately steep slope, with To $=-5.5$ (with eigenvectors for modes A, B, and $\mathrm{C}$ shown in Fig. 6). The contributions to the integrands in these expressions, for the three dominant modes, are shown in Fig. 9 and the integrals over all space are given in Table 1. The absolute magnitude of the eigenvector (containing the perturbation variables) is arbitrary since the calculation is based on solutions to the linear problem. But the relative size between different variables is dynamically consistent, so comparing the two expressions will give the relative importance of baroclinic (MAPE $\rightarrow$ EAPE) to barotropic (MKE $\rightarrow$ EKE) conversion.

Starting with mode A, we see that eddy $v$-momentum fluxes $\overline{u_{1}^{\prime} v_{1}^{\prime}}$ in a small region west of the jet maximum are up the mean-flow velocity gradient, a behavior that would tend to sharpen the jet. However, the integral of this part is small and completely overwhelmed by downgradient momentum fluxes everywhere else. Therefore, for this
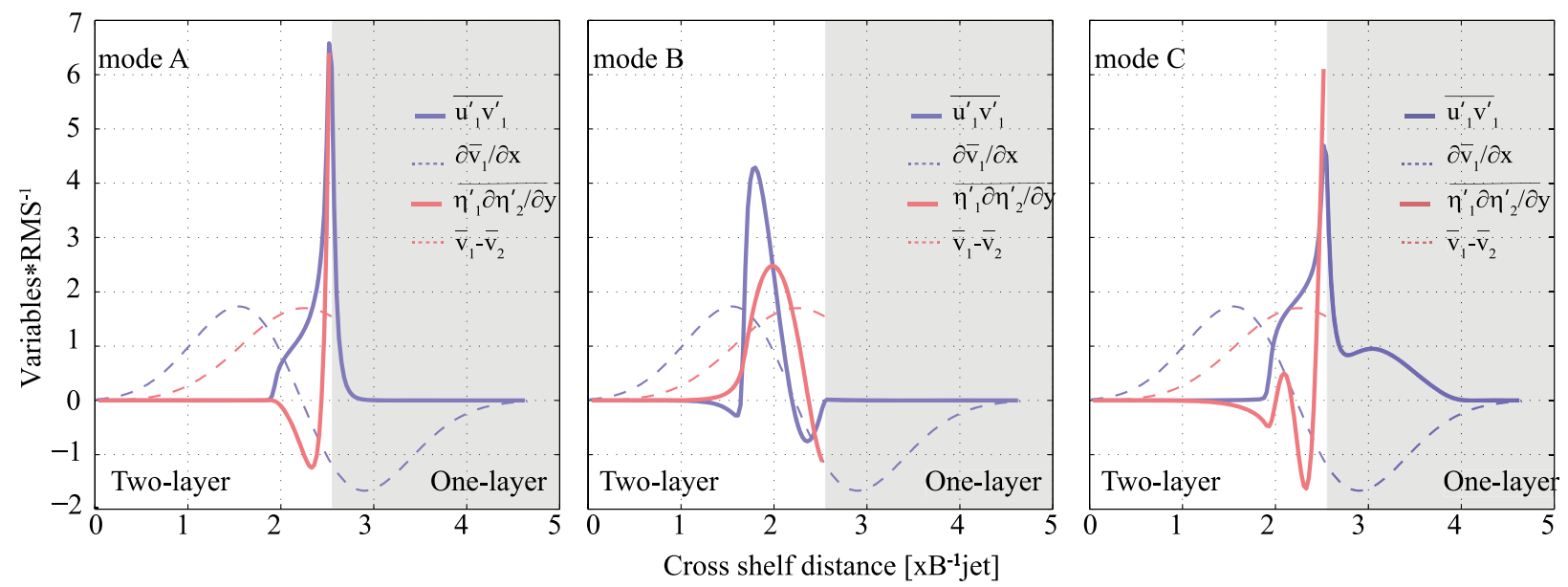

FIG. 9. The key quantities going into the energy transformation terms for the passive lower-layer configuration with To $=-5.5$. 
TABLE 1. The integrated energy transformations (arbitrary units) for the passive lower layer configuration with $\mathrm{To}=-5.5$.

\begin{tabular}{lccc}
\hline \hline & \multicolumn{3}{c}{ Modes } \\
\cline { 2 - 4 } $\begin{array}{c}\text { Energy } \\
\text { transformation }\end{array}$ & $\mathrm{A}$ & $\mathrm{B}$ & $\mathrm{C}$ \\
\hline$C_{\mathrm{MAPE} \rightarrow \text { EAPE }}$ & $2.6 \times 10^{-9}$ & $8.3 \times 10^{-8}$ & $6.7 \times 10^{-9}$ \\
$C_{\mathrm{MKE} \rightarrow \text { EKE }}$ & $1.3 \times 10^{-7}$ & $-4.8 \times 10^{-9}$ & $1.3 \times 10^{-7}$ \\
\hline
\end{tabular}

mode there is a net release of MKE to EKE, of magnitude $1.3 \times 10^{-7}$ (arbitrary units), consistent with barotropic instability (Table 1 ). The form drag $g \overline{\eta_{1}^{\prime} \partial \eta_{1}^{\prime} / \partial y}$ is both negative and positive and sums up to $2.6 \times 10^{-9}$. The mode thus extracts some MAPE but at a rate that is 50 times smaller than its extraction of MKE. Mode C shows some more horizontal structure but acts similarly in an integral sense (extracting 20 times more MKE than MAPE). For mode B the situation is reversed. The conversion terms are only nonzero seaward of the inflection point and give a clean picture of what goes on there. The eddy form drag is positive everywhere and adds up to $8.3 \times 10^{-8}$, whereas eddy momentum fluxes are almost entirely into the mean jet and about a factor 20 smaller $\left(-4.8 \times 10^{-9}\right)$. Therefore, mode $\mathrm{B}$ reflects pure baroclinic instability, extracting MAPE associated with the tilted isopycnal. Modes A and $\mathrm{C}$ extract both MKE and MAPE, but considering the relative magnitudes of conversion, it is safe to conclude that they primarily reflect barotropic instability.

\section{Discussion and conclusions}

The main finding of this study is that that the Norwegian Atlantic Current (NwAC), as it passes the steepest part of the Norwegian continental slope off the LofotenVesterålen islands, is both barotropically and baroclinically unstable. Baroclinic instability has been investigated earlier with linear QG vertical mode equations (Isachsen 2015), but this is the first assessment of unstable growth due to the lateral shear. Figure 4 suggests that baroclinic instability may dominate (have fastest growth rates) for relatively weak bottom slopes or, more specifically, for low ratios of the bottom to isopycnal slope (slope parameter To). But barotropic instability gets progressively more important and may even dominate for steeper bottom slopes. The same qualitative result was also found by Poulin et al. (2014) in a stability study of the coastal Bransfield Current in the Southern Ocean. The stronger suppression of baroclinic instability over steep bottom slopes may be intuitively understood from the fact that this process requires motion in the lower layer (where the topographic slope impacts the PV gradient), whereas lateral shear instability in the upper-layer jet does not.
Our study is limited in scope on many accounts. To begin with, in reality two currents are squeezed together by the converging isobaths off the Lofoten-Vesterålen islands: the NwAC and the Norwegian Coastal Current (NCC), which consists of buoyant coastal waters originating from the Baltic Sea and Norwegian river runoff (see schematics of currents in Fig. 1). The NCC flows shoreward of the NwAC, and the different hydrographic properties of the two water types do set up conditions for additional flow instabilities. Here, essentially, we have focused on the stability properties of the front that separates AW from basin waters offshore.

In extending the eigen problem to two dimensions, we have reduced the vertical resolution to two layers. This is, of course, an extreme simplification, and it is worth noting that the study of Isachsen (2015) found growth rates of baroclinic instability that were higher than those predicted by modified Eady or two-layer Phillips theory. What was observed (with the vertically high-resolution equations) was a tendency for internal PV gradients to suppress the bottom stabilization, an effect that requires at least three layers (see, e.g., Ikeda 1983). We intend to pursue three layers in future work but have here started with two SW layers as a simplest-possible model that can contain a lateral shear instability in the top layer.

We have ignored the possible influence of bottom friction on instability. Both Lin and Pierrehumbert (1988) and Stipa (2004) found that adding a bottom Ekman layer reduces growth rates of baroclinic instability as well as forces growth toward lower wavenumbers. Both, however, conclude that bottom friction is unable to shut down growth entirely. The implication to our problem here is that bottom friction could reduce growth rates of the baroclinic instability mode (mode B) preferentially over the upper-layer lateral shear modes (A and C), but that all modes would still exist.

Our very simplified geometry, both of bottom topography and background flow, also restricts the applicability of the quantitative results to the real situation off the Lofoten-Vesterålen islands. The simplified geometry allowed us to systematically investigate the qualitative effect of the bottom slope (via slope parameter To) on both types of instability. But other parts of parameter space were not investigated, for example, cases where the upper-layer jet is not centered over the isopycnal incrop. An upper-layer relative vorticity gradient is able to both enhance and suppress baroclinic instability itself depending on its horizontal position relative to the baroclinic shear (McIntyre 1970; Mechoso and Sinton 1981; James 1988).

Finally, we have only calculated growth rates based on annual-mean fields. As mentioned in the introduction, conditions vary substantially between summer and winter. 
Upper-layer flow speeds can be nearly twice as high in winter compared to summer. And since both the location and width of the jet are locked by topography, one can expect that lateral shears are stronger in winter, tending to favor barotropic instability. Plots of summer and winter conditions (not shown) reveal that the lateral density gradient increases in winter while the vertical stratification decreases (due to cooling). So winters are associated with steeper isopycnal slopes and hence smaller (less negative) To parameters. One would infer from this that baroclinic instability is favored in winter while barotropic instability is favored in summer. But such a conclusion needs to be qualified by the above observation that the lateral velocity shear is also enhanced during winter. A quantitative study of summer versus winter conditions is, however, beyond the scope of this initial study.

Perhaps the most serious technical limitation has been in the treatment of the eastern boundary condition for the lower layer, where the bounding isopycnals runs into the continental slope. As done in several earlier studies, we have assumed $u_{2}^{\prime}=0$ there, a limit only strictly applicable to a vertical eastern wall. The choice was made as a matter of convenience since the correct linearized boundary condition, derived in appendix A, does not seem to permit solving by eigen methods. We tested the sensitivity to this choice by comparison with a Robintype boundary condition in the appendix. The results, luckily, suggest that the fundamental findings presented here are robust with respect to the details of the lowerlayer boundary condition.

To summarize, our calculations have shown that both baroclinic and barotropic instability are sensitive to the continental slope steepness, at least in a linearized two-layer SW model. Our mode B, representing pure baroclinic instability, essentially followed the expected behavior outlined by two-layer QG theory. That modes $\mathrm{A}$ and $\mathrm{C}$, those believed to reflect barotropic instability, also show a similar response to variations in slope steepness is more surprising. But we have seen that these modes do have velocity signatures in the lower layer as well. Even if lower-layer perturbation velocities are weaker than those in the top layer (as we expect), it is likely that their interaction with the bottom topography impacts growth rates. Regardless of such details, the calculations give strong indication that the NwAC is also barotropically unstable off the Lofoten-Vesterålen islands, with growth rates that are comparable to or even higher than those of baroclinic instability over the steepest parts of the continental slope. This should have an impact on how we think of and model cross-shelf exchanges in this ocean region.
Acknowledgments. This work was supported under the ARCTOS LoVe MarinEco project financed by Equinor (Grant 4502610853) and by the NorSEE, ProVoLo and FjoCon projects funded by The Research Council of Norway (Grants 221780, 250784, and 225218).

\section{APPENDIX A}

\section{The Kinematic Boundary Condition at the Incrop Point}

We here take a closer look at the kinematic condition at the point where the interface intersects or incrops on the continental slope. This incrop point will hereafter be referred to as IP, and we let it be situated at $x=x_{0}$, as depicted in Fig. A1. For the discussion here we let the $x$ axis run through the IP of the undisturbed interface. The height of the mean interface (in thermal wind balance with the mean flow) is then given by $z=\bar{\eta}_{2}(x)$ and the bottom is at $z=-H_{2}(x)$. At the IP we have by definition $\bar{\eta}_{2}\left(x_{0}\right)=0$. Since the interface and the continental shelf are material surfaces, the exact kinematic boundary conditions are

$$
\begin{aligned}
& \frac{D\left(z-\eta_{2}\right)}{D t}=0, \quad z=\eta_{2}, \\
& \frac{D\left(z+H_{2}\right)}{D t}=0, \quad z=-H_{2},
\end{aligned}
$$

where $D / D t$ denotes the material derivative. Introducing perturbations and mean values [see (9)], the linearized versions of (A1) and (A2) become

$$
\begin{aligned}
& w_{2}^{\prime}=\frac{\partial \eta_{2}^{\prime}}{\partial t}+u_{2}^{\prime} \frac{d \bar{\eta}_{2}}{d x}+\bar{v}_{2} \frac{\partial \eta_{2}^{\prime}}{\partial y}, \quad z=\bar{\eta}_{2}, \\
& w_{2}^{\prime}=-u_{2}^{\prime} \frac{d H_{2}}{d x}, \quad z=-H_{2} .
\end{aligned}
$$

At the IP $x=x_{0}$ the two vertical velocities are equal, giving

$$
\frac{\partial \eta_{2}^{\prime}}{\partial t}=-u_{2}^{\prime} \frac{d \bar{h}_{2}}{d x}-\bar{v}_{2} \frac{\partial \eta_{2}^{\prime}}{\partial y}, \quad z=h_{2}\left(x_{0}\right)
$$

Note that this is equivalent to the lower-layer continuity equation (15) when the mean layer depth there goes to zero (at the intersect point). Rewriting to solve for $u_{2}^{\prime}$ gives

$$
-u_{2}^{\prime} \frac{d \bar{h}_{2}}{d x}=\frac{\partial \eta_{2}^{\prime}}{\partial t}+\bar{v}_{2} \frac{\partial \eta_{2}^{\prime}}{\partial y}, \quad z=h_{2}\left(x_{0}\right)
$$

or, for the assumed wave solution, 


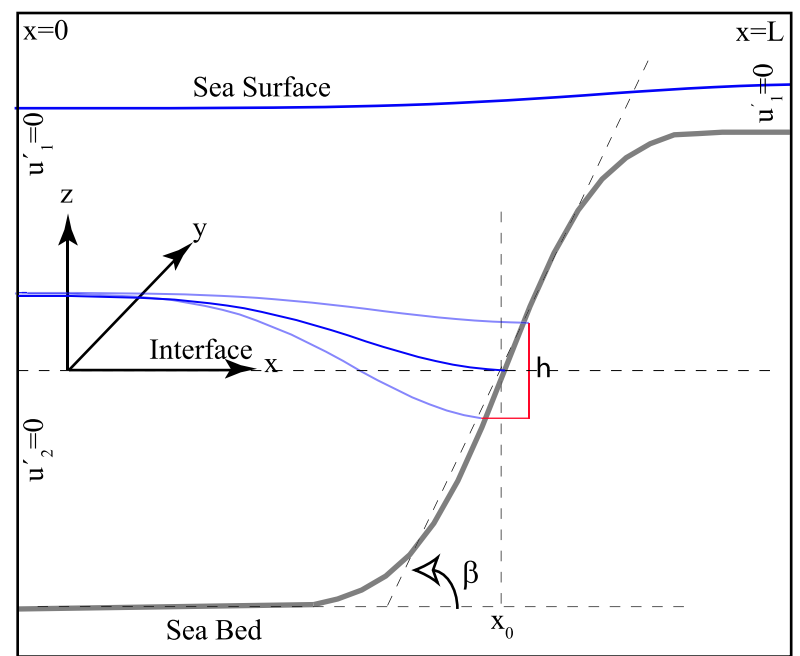

FIG. A1. Sketch depicting the coordinate system used to discuss the kinematic boundary condition associated with the isopycnal incrop.

$$
-\hat{u}_{2} \frac{d \bar{h}_{2}}{d x}=-i \omega \hat{\eta}_{2}+\bar{v}_{2} i l \hat{\eta}_{2}, \quad z=h_{2}\left(x_{0}\right),
$$

where $\bar{h}_{2}=H_{2}+\bar{\eta}_{2}$. This (rather than $\hat{u}_{2}=0$ ) constitutes the correct linearized boundary condition in the lower layer at the incrop point.

As it turns out, the implementation of the correct boundary condition is nontrivial on our staggered grid. By letting the incrop point fall on a $u$ point, say the $J$ th $u$ point, there is no problem in the lower-layer continuity equation. Since, by definition, $\bar{h}_{2 J}^{(u)}=0$ there, the discretized form of the equation for $\hat{\eta}_{2, J}$ (as well as its substitution into the equation for $\hat{\eta}_{1, J}$ ) simply becomes

$$
\begin{aligned}
& i l\left(\bar{v}_{2, J} \hat{\eta}_{2, J}+\bar{h}_{2, J} \hat{v}_{2, J}\right)-\frac{1}{\delta x}\left[\left(\frac{\bar{h}_{2, J-1}+\bar{h}_{2, J}}{2}\right) \hat{u}_{2, J-1}\right] \\
& \quad=i \omega \hat{\eta}_{2, J} .
\end{aligned}
$$

But the equation for $\hat{v}_{2, J}$ reads

$$
\begin{aligned}
& i l\left(g \hat{\boldsymbol{\eta}}_{1, J}+g^{\prime} \hat{\eta}_{2, J}+\bar{v}_{2, J} \hat{v}_{2, J}\right)+\left(\frac{\hat{u}_{2, J-1}+\hat{u}_{2, J}}{2}\right)\left(f+\frac{d \bar{v}_{2}}{d x}{ }_{J}\right) \\
& \quad=i \omega \hat{v}_{2, J},
\end{aligned}
$$

and this requires an expression for $\hat{u}_{2, J}$, that is, $\hat{u}_{2}$ at the incrop point. Inserting a discretized form of (A7) here will introduce the time derivative of $\eta_{2}$, making solution by eigen methods formally impossible. The additional time derivative may potentially be approximated by evaluating it at the $J$ th $\eta / v$ point, that is, half a grid point to the west of the incrop. But it is unclear whether the operation, which involves inserting the lower-layer continuity equation (with nonzero $\bar{h}_{2}$ ) into the boundary condition, is formally acceptable.

Here we will instead investigate how sensitive the fastest-growing unstable modes are to the treatment of the lower-layer boundary condition (and, specifically, its insertion into the last $\hat{v}_{2}$ equation). Two extreme behaviors of $\hat{u}_{2}$ can be expected at the incrop point, namely, $\hat{u}_{2}=0$ (relevant for an infinite bottom slope, i.e., a vertical wall) and $d \hat{u}_{2} / d x=0$ (to be expected in the limit of a very weak slope). Here we will compare the growth obtained when assuming each of these two extremes and also some behavior in between. We do this by implementing a Robintype boundary condition (Gustafson 1998),

$$
\hat{u}_{2}+L \frac{d \hat{u}_{2}}{d x}=0, \quad x=x_{0},
$$

where the parameter $L$ determines the relative importance of the two constraints. It seems natural to relate this to the bottom slope angle $\beta$ (see Fig. A2). For a near vertical wall $(\beta \rightarrow \pi / 2)$, we should have $L \rightarrow 0$ to obtain $\hat{u}_{2} \rightarrow 0$. For a very small bottom slope $(\beta \rightarrow 0)$, we should have $L \rightarrow \infty$ such that $d \hat{u}_{2} / d x \rightarrow 0$. This makes sense, since in the last case there is little hindrance for a particle associated with the perturbation to move horizontally back and forth around the IP.

To express these limiting cases and the transition between them explicitly, we set $L=\hat{L} \delta x \cot \beta$, where $\hat{L}$ is a free parameter (independent of $\beta$ ). Then our boundary condition becomes

$$
\hat{u}_{2}+\hat{L} \delta x \cot \beta \frac{d \hat{u}_{2}}{d x}=0, \quad x=x_{0},
$$

or, when discretized,

$$
\hat{u}_{2, J}+\hat{L} \cot \beta\left(\hat{u}_{2, J}-\hat{u}_{2, J-1}\right)=0,
$$

where we have had to evaluate the derivative at the last $\eta / v$ point. In terms of $\hat{u}_{2, J}$ [the quantity that needs to be substituted into (A9)]:

$$
\hat{u}_{2, J}=\frac{\hat{L} \cot \beta}{1+\hat{L} \cot \beta} \hat{u}_{2, J-1} .
$$

So for a given value of parameter $\hat{L}, \hat{u}_{2, J}$ varies smoothly between zero and $\hat{u}_{2, J-1}$ depending on the bottom slope.

We investigated the sensitivity of our calculations to the boundary condition by recalculating the results shown in Fig. 3b (bottom slope parameter To $=-4.2$ ), but now for three different values of $\hat{L}$. For the specific bottom slope of this problem, choosing $\hat{L}=1.0 \times 10^{-4}$ essentially gives $\hat{u}_{2}=0$ at the incrop point while $\hat{L}=1.0$ gives $d \hat{u}_{2} / d x=0$, and finally $\hat{L}=1.5 \times 10^{-2}$ provides an intermediate condition. Three different sets of markers 


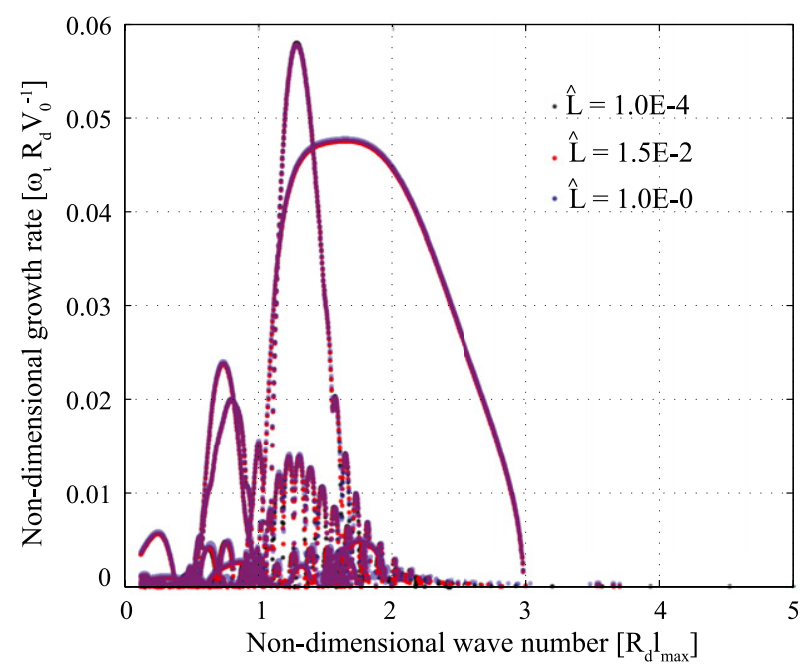

FIG. A2. Sensitivity of unstable growth to free parameter $\hat{L}$ in the Robin-type boundary condition for To $=-5.0$. Blue, green, and red dots represent growth rates for $\hat{L}=10^{-4}\left(\hat{u}_{2, J} \simeq 0\right), \hat{L}=10^{-2}$, and $\hat{L}=1\left(\hat{u}_{2, J} \simeq \hat{u}_{2, J-1}\right)$, respectively.

have been used in the plot but the growth rates of the three dominant modes are indistinguishable. The same result was found for other To values. Hence, we conclude that the fastest-growing unstable modes in the problem studied here are not sensitive to the exact treatment of the difficult boundary condition at the incrop point. Whether this result extends to other configurations will be the topic of another study.

\section{APPENDIX B}

\section{Energy Transformation}

The perturbation or "eddy" energy equations for the linear stability problem, valid to second order in perturbation amplitudes, can be derived from the linearized shallow water equations (10)-(15). To find expressions for EKE, we multiply the layer 1 and layer $2 u$-momentum equations by $u_{1}^{\prime}$ and $u_{2}^{\prime}$, respectively, and the two $v$-momentum equations by $v_{1}^{\prime}$ and $v_{2}^{\prime}$. Adding the resulting expressions for each layer gives

$$
\begin{aligned}
& \frac{\partial\left(e k_{1}\right)}{\partial t}+\frac{\partial\left(\bar{v}_{1} e k_{1}\right)}{\partial y}+u_{1}^{\prime} v_{1}^{\prime} \frac{\partial \bar{v}_{1}}{\partial x}=-g \mathbf{u}_{1}^{\prime} \cdot \nabla \eta_{1}^{\prime}, \quad \text { and } \\
& \frac{\partial e k_{2}}{\partial t}+\frac{\partial\left(\bar{v}_{2} e k_{2}\right)}{\partial y}+u_{2}^{\prime} v_{2}^{\prime} \frac{\partial \bar{v}_{2}}{\partial x}=-g \mathbf{u}_{2}^{\prime} \cdot \nabla \eta_{1}^{\prime}-g^{\prime} \mathbf{u}_{2}^{\prime} \cdot \nabla \eta_{2}^{\prime},
\end{aligned}
$$

where we have defined the EKE densities $e k_{1}=\left(u_{1}^{\prime 2}+\right.$ $\left.v_{1}^{\prime 2}\right) / 2$ and $e k_{2}=\left(u_{2}^{\prime 2}+v_{2}^{\prime 2}\right) / 2$. Integrating vertically over mean layer thicknesses $\bar{h}_{1}=H_{1}+\bar{\eta}_{1}-\bar{\eta}_{2}$ and $\bar{h}_{2}=$ $\mathrm{H}_{2}+\bar{\eta}_{2}$ yields

$\frac{\partial\left(\bar{h}_{1} e k_{1}\right)}{\partial t}+\frac{\partial\left(\bar{h}_{1} \bar{v}_{1} e k_{1}\right)}{\partial y}+\bar{h}_{1} u_{1}^{\prime} v_{1}^{\prime} \frac{\partial \bar{v}_{1}}{\partial x}=-g \bar{h}_{1} \mathbf{u}_{1}^{\prime} \cdot \nabla \eta_{1}^{\prime}, \quad$ and

$$
\begin{aligned}
& \frac{\partial\left(\bar{h}_{2} e k_{2}\right)}{\partial t}+\frac{\partial\left(\bar{h}_{2} v_{2} e k_{2}\right)}{\partial y}+\bar{h}_{2} u_{2}^{\prime} v_{2} \frac{\partial \bar{v}_{2}}{\partial x}=-g \bar{h}_{2} \mathbf{u}_{2}^{\prime} \cdot \nabla \eta_{1}^{\prime} \\
& -g^{\prime} \bar{h}_{2} \mathbf{u}_{2}^{\prime} \cdot \nabla \eta_{2}^{\prime},
\end{aligned}
$$

or, after rearranging the pressure gradient terms,

$$
\begin{aligned}
& \frac{\partial\left(\bar{h}_{1} e k_{1}\right)}{\partial t}+\frac{\partial\left(\bar{h}_{1} \bar{v}_{1} e k_{1}\right)}{\partial y}+\bar{h}_{1} u_{1}^{\prime} v_{1}^{\prime} \frac{\partial \bar{v}_{1}}{\partial x}=-\nabla \cdot\left(g \bar{h}_{1} \mathbf{u}_{1}^{\prime} \eta_{1}^{\prime}\right) \\
& \quad+g \eta_{1}^{\prime} \nabla \cdot\left(\mathbf{u}_{1}^{\prime} \bar{h}_{1}\right), \text { and } \\
& \frac{\partial\left(\bar{h}_{2} e k_{2}\right)}{\partial t}+\frac{\partial\left(\bar{h}_{2} \bar{v}_{2} e k_{2}\right)}{\partial y}+\bar{h}_{2} u_{2}^{\prime} v_{2} \frac{\partial \bar{v}_{2}}{\partial x}=-\nabla \cdot\left(g \bar{h}_{2} \mathbf{u}_{2}^{\prime} \eta_{1}^{\prime}\right) \\
& \quad+g \eta_{1}^{\prime} \nabla \cdot\left(\mathbf{u}_{2}^{\prime} \bar{h}_{2}\right)-\nabla \cdot\left(g^{\prime} \bar{h}_{2} \mathbf{u}_{2}^{\prime} \eta_{2}^{\prime}\right)+g^{\prime} \eta_{2}^{\prime} \nabla \cdot\left(\mathbf{u}_{2}^{\prime} \bar{h}_{2}\right) .
\end{aligned}
$$

The EAPE equations are found by multiplying layer 1 and layer 2 thickness equations by $g \eta_{1}^{\prime}$ and $g^{\prime} \eta_{2}^{\prime}$, respectively. This gives

$$
\begin{aligned}
\frac{\partial}{\partial t}\left(g \frac{\eta_{1}^{\prime 2}}{2}\right)= & -g \eta_{1}^{\prime} \nabla \cdot\left(\mathbf{u}_{2}^{\prime} \bar{h}_{2}\right)-g \eta_{1}^{\prime} \nabla \cdot\left(\mathbf{u}_{1}^{\prime} \bar{h}_{1}\right) \\
& -\frac{\partial}{\partial y}\left(g \bar{v}_{1} \frac{\eta_{1}^{\prime 2}}{2}\right)+g\left(\bar{v}_{1}-\bar{v}_{2}\right) \eta_{1}^{\prime} \frac{\partial}{\partial y} \eta_{2}^{\prime}, \text { and }
\end{aligned}
$$

$$
\frac{\partial}{\partial t}\left(g^{\prime} \frac{\eta_{2}^{\prime 2}}{2}\right)=-g^{\prime} \eta_{2}^{\prime} \nabla \cdot\left(\mathbf{u}_{2}^{\prime} \bar{h}_{2}\right)-\frac{\partial}{\partial y}\left(g^{\prime} \bar{v}_{2} \frac{\eta_{2}^{\prime 2}}{2}\right)
$$

We note three terms that show up both in the EKE and EAPE equations, involving divergences of the layer transports. These represent local transfers between EKE and EAPE that cancel when we add the equations to obtain an expression for total eddy energy (Cushman-Roisin and Beckers 2011, chapter 12). We form such a total eddy energy equation and then, finally, integrate over all space. The integral in $y$ is over an integer number of wavelengths and will be denoted by $(\ldots)$. The integral in $x$ is from $x=0$ to $x=L$ (or to the incrop point for layer 2 variables) and will be denoted $\langle(\ldots)\rangle$. The periodic boundary conditions in $y$ and the $u^{\prime}=0$ boundary conditions in $x$ cause the flux divergence terms to vanish, and we end up with 


$$
\begin{aligned}
\frac{d}{d t}\langle\mathrm{EKE}+\mathrm{EAPE}\rangle= & -\left\langle\bar{h}_{1} \overline{u_{1}^{\prime} v_{1}^{\prime}} \frac{\partial \bar{v}_{1}}{\partial x}\right\rangle-\left\langle\bar{h}_{2} \overline{u_{2}^{\prime} v_{2}^{\prime}} \frac{\partial \bar{v}_{2}}{\partial x}\right\rangle \\
& +\left\langle g\left(\bar{v}_{1}-\bar{v}_{2}\right) \overline{\eta_{1}^{\prime} \frac{\partial \eta_{2}^{\prime}}{\partial y}}\right\rangle .
\end{aligned}
$$

Hence, for the linearized stability problem the time rate of change of total eddy kinetic plus eddy potential energy of the system is governed by three terms:

$$
\begin{aligned}
C_{\mathrm{MKE}_{1} \rightarrow \mathrm{EKE}_{1}} & =-\left\langle\bar{h}_{1} \overline{u_{1}^{\prime} v_{1}^{\prime}} \frac{\partial \bar{v}_{1}}{\partial x}\right\rangle, \\
C_{\mathrm{MKE}_{2} \rightarrow \mathrm{EKE}_{2}} & =-\left\langle\bar{h}_{2} \overline{u_{2}^{\prime} v_{2}^{\prime}} \frac{\partial \bar{v}_{2}}{\partial x}\right\rangle, \quad \text { and } \\
C_{\mathrm{MAPE} \rightarrow \mathrm{EAPE}} & =\left\langle g\left(\bar{v}_{1}-\bar{v}_{2}\right) \overline{\eta_{1}^{\prime} \frac{\partial \eta_{2}^{\prime}}{\partial y}}\right\rangle .
\end{aligned}
$$

The first two terms release mean kinetic energy from layer 1 and 2 by an eddy momentum flux out of the mean jet while the third term releases mean available potential energy via a form drag acting on the interface between the two layers (Vallis 2017, chapter 3). For perturbations that are geostrophic this last term may be rewritten (after one integration by parts) as $C_{\mathrm{MAPE} \rightarrow \mathrm{EAPE}}=-\left\langle g^{\prime} u_{1}^{\prime} \eta_{2}^{\prime} \partial \bar{\eta}_{2} / \partial x\right\rangle$, representing an eddy bolus volume flux in the top layer that tends to flatten the isopycnal.

\section{REFERENCES}

Albretsen, J., A. K. Sperrevik, A. Staalstrøm, A. D. Sandvik, F. Vikeb $\varnothing$, and L. Asplin, 2011: Norkyst-800 Report No. 1: User manual and technical descriptions. Tech. Rep. 2/2011, Fisken og Havet, 46 pp., https://www.imr.no/filarkiv/2011/07/ fh_2-2011_til_web.pdf/nb-no.

Andersson, M., K. A. Orvik, J. H. LaCasce, I. Koszalka, and C. Mauritzen, 2011: Variability of the Norwegian Atlantic Current and associated eddy field from surface drifters. J. Geophys. Res., 116, C08032, https://doi.org/10.1029/2011JC007078.

Blumsack, S. L., and P. J. Gierasch, 1972: Mars: The effects of topography on baroclinic instability. J. Atmos. Sci., 29, 1081-1089, https://doi.org/10.1175/1520-0469(1972)029<1081: MTEOTO $>2.0 . \mathrm{CO} ; 2$.

Butler, K. M., and B. F. Farrell, 1992: Three-dimensional optimal perturbations in viscous shear flow. Phys. Fluids, 4A, 16371650, https://doi.org/10.1063/1.858386.

Cushman-Roisin, B., and J.-M. Beckers, 2011: Introduction to Geophysical Fluid Dynamics: Physical and Numerical Aspects. 2nd ed. International Geophysics Series, Vol. 101, Academic Press, 875 pp.

Drange, H., T. Dokken, T. Furevik, R. Gerdes, and W. Berger, Eds., 2005: The Nordic Seas: An Integrated Perspective. Geophys. Monogr., Vol. 158, Amer. Geophys. Union, https:// doi.org/10.1029/GM158.

Gustafson, K., 1998: Domain decomposition, operator trigonometry, Robin condition. Contemp. Math., 218, 432-437, https:// doi.org/10.1090/conm/218/3039.
Horn, W. and F. Schott, 1976: Measurements of stratification and currents at the Norwegian continental slope. "Meteor" Forschungsergeb., Reihe A, 18, 23-63.

Ikeda, M., 1983: Linear instability of a current flowing along a bottom slope using a three-layer model. J. Phys. Oceanogr., 13, 208-223, https://doi.org/10.1175/1520-0485(1983)013<0208: LIOACF $>2.0 . \mathrm{CO} ; 2$.

Isachsen, P. E., 2015: Baroclinic instability and the mesoscale eddy field around the Lofoten Basin. J. Geophys. Res. Oceans, 120, 2884-2903, https://doi.org/10.1002/2014JC010448.

— , and O. A. Nøst, 2012: The air-sea transformation and residual overturning circulation within the Nordic Seas. J. Mar. Res., 70, 31-68, https://doi.org/10.1357/002224012800502372.

_ C. Mauritzen, and H. Svendsen, 2007: Dense water formation in the Nordic Seas diagnosed from sea surface buoyancy fluxes. Deep-Sea Res. I, 54, 22-41, https://doi.org/10.1016/ j.dsr.2006.09.008.

_ I. Koszalka, and J. H. LaCasce, 2012: Observed and modeled surface eddy heat fluxes in the eastern Nordic Seas. J. Geophys. Res., 117, C08020, https://doi.org/10.1029/ 2012JC007935.

James, I. N., 1988: Suppression of baroclinic instability in horizontally sheared flows. J. Atmos. Sci., 44, 3710-3720, https:// doi.org/10.1175/1520-0469(1987)044<3710:SOBIIH>2.0.CO;2.

Käse, R. H., N. Serra, A. Köhl, and D. Stammer, 2009: Mechanisms for the variability of dense water pathways in the Nordic Seas. J. Geophys. Res., 114, C01013, https://doi.org/10.1029/ 2008JC004916.

Koszalka, I., J. LaCasce, M. Andersson, K. Orvik, and C. Mauritzen, 2011: Surface circulation in the Nordic Seas from clustered drifters. Deep-Sea Res. I, 58, 468-485, https:// doi.org/10.1016/j.dsr.2011.01.007.

,-- , and C. Mauritzen, 2013: In pursuit of anomalies: Analyzing the poleward transport of Atlantic water with surface drifters. Deep-Sea Res. II, 85, 96-108, https://doi.org/10.1016/ j.dsr2.2012.07.035.

Lin, S.-J., and R. T. Pierrehumbert, 1988: Does Ekman friction suppress baroclinic instability? J. Atmos. Sci., 45, 2920-2933, https://doi.org/10.1175/1520-0469(1988)045<2920:DEFSBI> 2.0.CO;2.

Mauritzen, C., 1996: Production of dense overflow waters feeding the North Atlantic across the Greenland-Scotland Ridge. Part 1: Evidence for a revised circulation scheme. Deep-Sea Res. I, 43, 769-806, https://doi.org/10.1016/0967-0637(96)00037-4.

McIntyre, M. E., 1970: On the nonseparable baroclinic parallel flow instability problem. J. Fluid Mech., 40, 273-306, https:// doi.org/10.1017/S0022112070000174.

Mechoso, C. R., 1980: Baroclinic instability of flows along sloping boundaries. J. Atmos. Sci., 37, 1393-1399, https://doi.org/ 10.1175/1520-0469(1980)037<1393:BIOFAS >2.0.CO;2.

_ , and D. M. Sinton, 1981: Instability of baroclinic flows with horizontal shear along topography. J. Phys. Oceanogr., 11, 813-821, https://doi.org/10.1175/1520-0485(1981)011<0813: IOBFWH $>2.0 . \mathrm{CO} ; 2$.

Mork, K. A., and Ø. Skagseth, 2010: A quantitative description of the Norwegian Atlantic Current by combining altimetry and hydrography. Ocean Sci., 6, 901-911, https://doi.org/10.5194/os-6-901-2010.

Mysak, L. A., and F. Schott, 1977: Evidence for baroclinic instability of the Norwegian Current. J. Geophys. Res., 82, 20872095, https://doi.org/10.1029/JC082i015p02087.

Nurser, A. J. G., and S. Bacon, 2014: The Rossby radius in the Arctic Ocean. Ocean Sci., 10, 967-975, https://doi.org/10.5194/ os-10-967-2014. 
Olbers, D., J. Willebrand, and C. Eden, 2012: Ocean Dynamics. Springer, $703 \mathrm{pp}$.

Orvik, K. A., and P. P. Niiler, 2002: Major pathways of Atlantic water in the northern North Atlantic and Nordic Seas toward Arctic. Geophys. Res. Lett., 29, 1986, https://doi.org/10.1029/2002GL015002.

- and $\varnothing$. Skagseth, 2003: The impact of the wind stress curl in the North Atlantic on the Atlantic inflow to the Norwegian Sea toward the Arctic. Geophys. Res. Lett., 30, 1884, https:// doi.org/10.1029/2003GL017932.

Poulain, P.-M., A. Warn-Varnas, and P. P. Niiler, 1996: Near-surface circulation of the Nordic Seas as measured by Lagrangian drifters. J. Geophys. Res., 101, 18237-18258, https://doi.org/10.1029/96JC00506.

Poulin, F. J., M. Stegner, A. Hernánzez-Arencibia, A. MarreroDíaz, and P. Sangrà, 2014: Steep shelf stabilization of the Coastal Bransfield Current: Linear stability analysis. J. Phys. Oceanogr., 44, 714-732, https://doi.org/10.1175/JPO-D-13-0158.1.

Raj, R., L. Chafik, J. E. Nilsen, T. Eldevik, and I. Halo, 2015: The Lofoten Vortex of the Nordic Seas. Deep-Sea Res. I, 96, 1-14, https://doi.org/10.1016/j.dsr.2014.10.011.

Skagseth, O., K. A. Orvik, and T. Furevik, 2004: Coherent variability of the Norwegian Atlantic Slope Current derived from TOPEX/ERS altimeter data. Geophys. Res. Lett., 31, L14304, https://doi.org/10.1029/2004GL020057.

Skjoldal, H. R., Ed., 2004: The Norwegian Sea Ecosystem. Tapir Academic Press, 559 pp.
Søiland, H., and T. Rossby, 2013: On the structure of the Lofoten Basin eddy. J. Geophys. Res. Oceans, 118, 4201-4212, https:// doi.org/10.1002/jgrc.20301.

Spall, M. A., 2010: Non-local topographic influences on deep convection: An idealized model for the Nordic Seas. Ocean Modell., 32, 72-85, https://doi.org/10.1016/j.ocemod.2009.10.009.

Stipa, T., 2004: On the sensitivity of coastal quasigeostrophic edge wave interaction to bottom boundary characteristics: Possible implications for eddy parameterizations. ArXiv, https://arxiv.org/ abs/physics/0401119.

Teigen, S. H., F. Nilsen, and B. Gjevik, 2010: Barotropic instability in the West Spitsbergen Current. J. Geophys. Res., 115, C07016, https://doi.org/10.1029/2009JC005996.

R. Skogseth, B. Gjevik, and A. Beszczynska-Möller, 2011: Baroclinic instability in the West Spitsbergen Current. J. Geophys. Res., 116, C07012, https://doi.org/10.1029/ 2011JC006974.

Trodahl, M., and P. E. Isachsen, 2018: Topographic influence on baroclinic instability and the mesoscale eddy field in the northern North Atlantic Ocean and the Nordic Seas. J. Phys. Oceanogr., 48, 2593-2607, https://doi.org/10.1175/ JPO-D-17-0220.1.

Vallis, G. K., 2017: Atmospheric and Oceanic Fluid Dynamics: Fundamentals and Large-Scale Circulation. 2nd ed. Cambridge University Press, 745 pp. 